\title{
Valsalva maneuver in detection of right-to-left shunt by transcranial Doppler
}

Sir: We read with great interest the study by Lange et al (Arq Neuropsiquiatr 2010;68:410-3) regarding the effect of valsalva maneuver (VM) in right-to-left shunt (RLS) detection by transcranial Doppler (TCD). We wish to highlight some relevant issues.

Trans-esophageal echocardiography (TEE) is considered the gold-standard for diagnosing RLS. Although, TEE provides anatomical details of RLS, the real-time travel of a thrombus has been observed rarely ${ }^{1}$. Limitations of TEE include the need of an expert echocardiographer, poor tolerance by patients and often insufficient VM due to sedation and endoscope in the throat. In contrast, TCD is reliable, performed without sedation and carries high sensitivity and specificity as compared to $\mathrm{TEE}^{2}$.

We disagree with the finding that the timing of VM does not influence the diagnosis of RLS. Considerable variations exist in the methodology for RLS detection, especially the timing of VM during the test performance. While VM has been performed simultaneously with the contrast injection in some studies ${ }^{3}$, others initiated it after a delay of 3-5 seconds ${ }^{2}$. Substantial hemodynamic changes occur during the "Strain-phase" (amplifies inter-atrial leftto-right pressure-gradient and counteracts RLS) as well as the "release-phase" (reverses pressure gradient with sudden increase in venous return and right atrial pressure that facilitate RLS) ${ }^{4}$. Furthermore, VM causes considerable reduction, and even complete stoppage, of flow in proximal large veins. Thus, the injection of saline-air contrast mixture during VM into a proximal vein (with elevated pressure and near-stagnant flow) does not seem logical. Injection during VM theoretically seems counterproductive since the high venous-pressure might destroy some of the "microbubbles" in "saline-air" contrast mixture. This assumes further significance as the microbubbles created during the vigorous shaking of the contrast mixture survive only for a short time. We believe that although, the timing of VM may not affect the detection of RLS, it might influence the functional grading of the shunt.

Body position during the diagnosis of RLS needs special consideration. TEE, usually performed with patient lying in left-lateral position, is met with an adverse environment for air microbubbles - to travel from right atrium (at higher level) into the left atrium (at lower level) - thus challenging the basic physical fundamentals! We have demonstrated previously that multiple injections of contrast mixture during TCD are safe and "functional-grades" of RLS varies with body positions as larger number of microbubbles are detected in sitting position $^{5}$. Larger studies with controlled conditions for various confounding factors are needed to develop a standardized protocol for RLS detection.

\section{REFERENCES}

1. Ozdogru I, Kaya MG, Dogan A, Inanc T, Yalcin A, Oguzhan A. Thrombus crossing through a patent foramen ovale. Int J Cardiol 2009;133:e55-56.

2. Jauss M, Kaps M, Keberle M, Haberbosch W, Dorndorf W. A comparison of transesophageal echocardiography and transcranial Doppler sonography with contrast medium for detection of patent foramen ovale. Stroke 1994; 25:1265-1267.

3. Klötzsch C, Janssen G, Berlit P. Transesophageal echocardiography and contrast-TCD in the detection of a patent foramen ovale: experiences with 111 patients. Neurology 1994;44:1603-1606.

4. Nishimura RA, Tajik AJ. The Valsalva maneuver and response revisited. Mayo Clin Proc 1986;61:211-217.

5. Lao AY, Sharma VK, Tsivgoulis G, Malkoff MD, Alexandrov AV, Frey JL. Effect of body positioning during transcranial Doppler detection of right-to-left shunts. Eur J Neurol 2007;14:1035-1039.

\author{
Prakash R. Paliwal, MRCP \\ Vijay K. Sharma, MRCP, RVT \\ Division of Neurology, National University Hospital, Singapore \\ Vijay Sharma - Division of Neurology, Department of \\ Medicine, National University Hospital, Singapore 119074 \\ E-mail-drvijay@singnet.com.sg
}

We have no competing interests with this manuscript 